\title{
Continuous Flow Controlled Synthesis of Gold Nanoparticles Using Pulsed Mixing Microfluidic System
}

\author{
Guojun Liu, ${ }^{1}$ Xuhao Yang, ${ }^{1}$ Yan Li, ${ }^{2}$ Zhigang Yang, ${ }^{1}$ Wen Hong, ${ }^{1}$ and JianFang Liu ${ }^{1}$ \\ ${ }^{1}$ College of Mechanical Science and Engineering, Jilin University, Changchun 130025, China \\ ${ }^{2}$ Department of Emergency, China-Japan Union Hospital, Jilin University, Changchun 130033, China \\ Correspondence should be addressed to Yan Li; zrlliyan@medmail.com.cn
}

Received 9 July 2015; Revised 6 October 2015; Accepted 20 October 2015

Academic Editor: Michele Iafisco

Copyright (C) 2015 Guojun Liu et al. This is an open access article distributed under the Creative Commons Attribution License, which permits unrestricted use, distribution, and reproduction in any medium, provided the original work is properly cited.

\begin{abstract}
To prepare the gold nanoparticles (AuNPs) with uniform sizes, fine morphology, and good monodispersity, a pulsed mixing microfluidic system based on PZT actuation was presented. The system includes PZT micropump and Y type micromixer. By adjusting voltage (entrance flow rate), pulsed frequency, phase, and other parameters, a variety of mixing modes can be achieved, so as to realize the controllable synthesis of nanoparticles in a certain range. By numerical simulation and analysis, the channel section size, entrance angle, and pulse frequency were optimized. Based on the optimized structure and working parameters, the test prototype has been manufactured in lab, and the related synthesis tests of AuNPs were carried out. The test results indicate that AuNPs with uniform morphology and good monodispersity can be synthesized using the system with the section size $(0.4 \mathrm{~mm} \times$ $0.4 \mathrm{~mm})$, the entrance channel angle $\left(60^{\circ}\right)$ under condition of the pulsed frequency $(300 \mathrm{~Hz})$, and the entrance flow rate $(4 \mathrm{~mL} / \mathrm{min})$. The average diameter and its standard deviation of AuNPs synthesized were $21.6 \mathrm{~nm}, 4.83 \mathrm{~nm}$, respectively. The research work above can be applied to the fields such as the controlled synthesis of noble metal nanoparticles, biomedicine, and microchemical system.
\end{abstract}

\section{Introduction}

Gold nanoparticles (AuNPs) have received tremendous growing attention during the recent few years because of their unique characteristics $[1-4]$ and have already found many kinds of applications in catalysis [5-7], sensing [810], diagnosis [11-13], biomedicine [1,14, 15], drug delivery [16-18], and so forth. In addition, it also has been applied to a chemical analysis with Localized Surface Plasmon (LSP) [19] and Surface Enhanced Raman Spectroscopy (SERS) [20, 21]; both the average size of the gold nanoparticles and the size distribution are critically important factors in such applications [22].

AuNPs which have been synthesized by various physical and chemical methods mainly include UV irradiation, laser ablation, plasma synthesis, citrate synthesis, turkevich method, wet chemical synthesis, and chemical reduction method [1]. The shapes of obtained AuNPs are multifarious, such as triangles [23, 24], nanorods [25], and stars [26]. The most of AuNPs synthesis process was achieved by reducing the gold salt in the water phase [27]. On the basis of the method, microfluidic mixer has been used for the synthesis of gold nanoparticles by scholars. The continuous flow methods using micromixers have been proven to be successful alternative methods with a possibility to synthesize large quantities with better control on the distribution, shape, and size of the particles $[28,29]$. However, some of reports still cannot realize a narrow distribution of particle size. The reason for that is the lack of rapid mixability at a laminar flow condition with a microchannel.

Mixing is a basic unit operation for chemical reactions and micromixing can extremely influence the selectivity, yield, and quality of final products in various chemical processes [30-32]. In this paper, a pulsed mixing microfluidic system for the synthesis of AuNPs is developed. Firstly, the mechanism of gold nanoparticles formation and pulsed micromixing is introduced. Secondly, the structure and working parameters of $\mathrm{Y}$ type micromixer are optimized after simulation analysis. Finally, the gold nanoparticles are prepared and the test results are discussed. 


\section{Mechanism of Controlled Synthesis of AuNPs}

2.1. Mechanism of AuNPs Formation. Based on chemical reduction mechanism, the liquid-phase synthesis method of AuNPs includes three processes, namely, precipitation of gold atoms, nucleation, and growth of crystal nuclei. The synthesis mechanism can be described by Figure 1 as follows. In the initial stage of mixing of the reducing agent (such as sodium citrate) and precursor (such as chloroauric acid), as the precursor is reduced, the concentration of gold atoms continues increasing (stage I: precipitation of gold atoms). When the concentration of gold atoms exceeds the critical supersaturation (stage II: nucleation), the gold atoms begin to gather and form crystal nuclei. Also, with the formation and growth of crystal nuclei, the concentration of gold atoms begins to decrease. After a large amount of gold atoms are consumed, the concentration of gold atoms gradually declines down below the critical supersaturation (stage III: growth of crystal nuclei), the number of crystal nuclei no longer increases, and the growth of nuclei will dominate the whole process. When the concentration of gold atoms decreases to the saturation level, crystal nuclei stop growing and the AuNPs are finally prepared. As can be seen from the above reaction processes, stages I and II are particularly important, whose mixing effect largely affects the amount and size uniformity of AuNPs prepared by the liquidphase synthesis method. To obtain the AuNPs with uniform morphology and small size deviation, besides the appropriate adjustment of ingredients proportion, concentration, and so forth of two-phase agents, it is crucial to regulate the mixed reaction efficiency. Only when the mixing effect can be controlled freely, will it become possible to realize the controlled synthesis of AuNPs. So the controllability of microfluidic reaction process is an important condition to achieve the AuNPs with uniform particle size, regular morphology.

2.2. Mechanism of Pulsed Micromixing. Pulsed micromixing is an active mixing method with high mixing efficiency [22, 33 ] and its working mechanism is shown in Figure 2. Pulsed micromixing system includes two functional parts, namely, driving source (PZT micropump) and Y type micromixer. Two PZT micropumps with same structure and working performance provide the mixing power for $\mathrm{Y}$ type micromixer. And two different kinds of fluids are pumped into the mixer with the changing flow rate, which fluctuates from high to low periodically by different frequency and phase. Therefore, the pulsing fluid fields will cause rapid stretching and folding of the fluid interfaces and then realize a variety of mixed modes, such as the laminar mixing, chaotic advection, and turbulent mixing. As illustrated in Figure 2, when two sine wave input signals have a phase difference of 180 degrees, solution A (such as chloroauric acid) and solution B (such as sodium citrate) achieve pulsating crossover type input in the micromixer. Thus, it increases the contact area and the contact opportunity of two kinds of liquid, shortens the mixing time, and improves the efficiency of mixing.

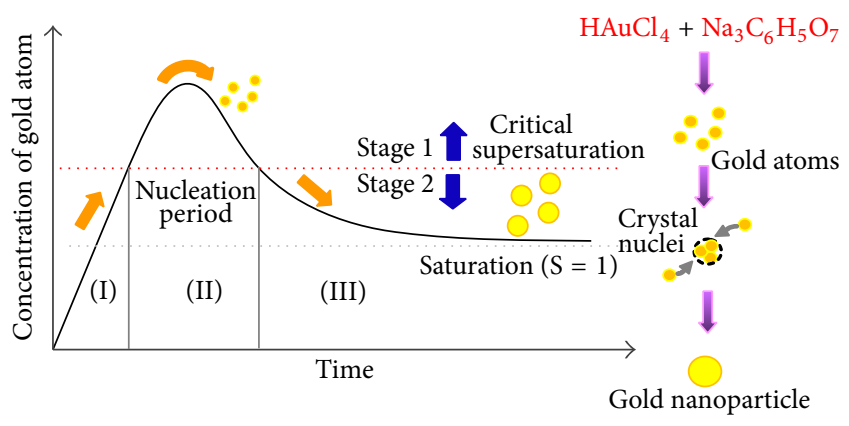

FIgURE 1: Schematic illustration of synthesis mechanism of gold nanoparticles.

\section{The Structure and Working Parameters Optimization of Y Type Micromixer}

3.1. Modeling of Y Type Micromixer. To optimize the structure of mixer, $Y$ type micromixer model is established as shown in Figure 3. Through the channel entrances 1 and 2, the two kinds of fluid are, respectively, pumped into the micromixer, complete mixing, or reaction in the mainstream channel of micromixer and then are output from the outlet. The main structure dimensions of mixer include entrance channel length $S$, angle between two flow entrances $\theta$, length of main mixing channel $L$, width of the channel $W$, and depth of flow channel $H$. The origin of coordinate system is set in the center of the junction of two entrance channels, with the $X$ direction along the mainstream channel.

3.2. Evaluation of Mixing Efficiency. To evaluate the mixing efficiency quantitatively, an important index parameter, namely, the degree of mixing $(\sigma)$, is defined [34]:

$$
\sigma=1.0-\frac{1}{c_{\infty}} \sqrt{\frac{\sum_{i=1}^{N}\left(c_{i}-c_{\infty}\right)^{2}}{N}},
$$

where $N$ is the number of sampling points inside the cross section, $c_{i}$ is the mass fraction at sampling point $i$, and $c_{\infty}$ is the optimal mixing mass fraction. The value of $\sigma$ should be initially zero and tend monotonically towards a large value during the mixing progresses. Larger $\sigma$ indicates better mixing efficiency. When the mixing degree $\sigma=0$, there is no mixture between two phases of fluid. When the mixing degree $\sigma=1$, two phases of fluid are in a real state of complete mixing.

\subsection{Optimization of the Structure Parameters}

3.3.1. Analysis of Effects of Section Size on the Mixing Effect. In experimental test, the flow rate of PZT micropump is fixed on a specified value (in this work, the flow rate of PZT micropump is $4 \mathrm{~mL} / \mathrm{min}$ ). At the entrance of mixer, the velocity amplitudes of two phase flow vary with size of section. In simulation analysis, four sets of structure parameters are selected as shown in Table 1, and the angle between the entrance channels is initially selected $90^{\circ}$. According to the 


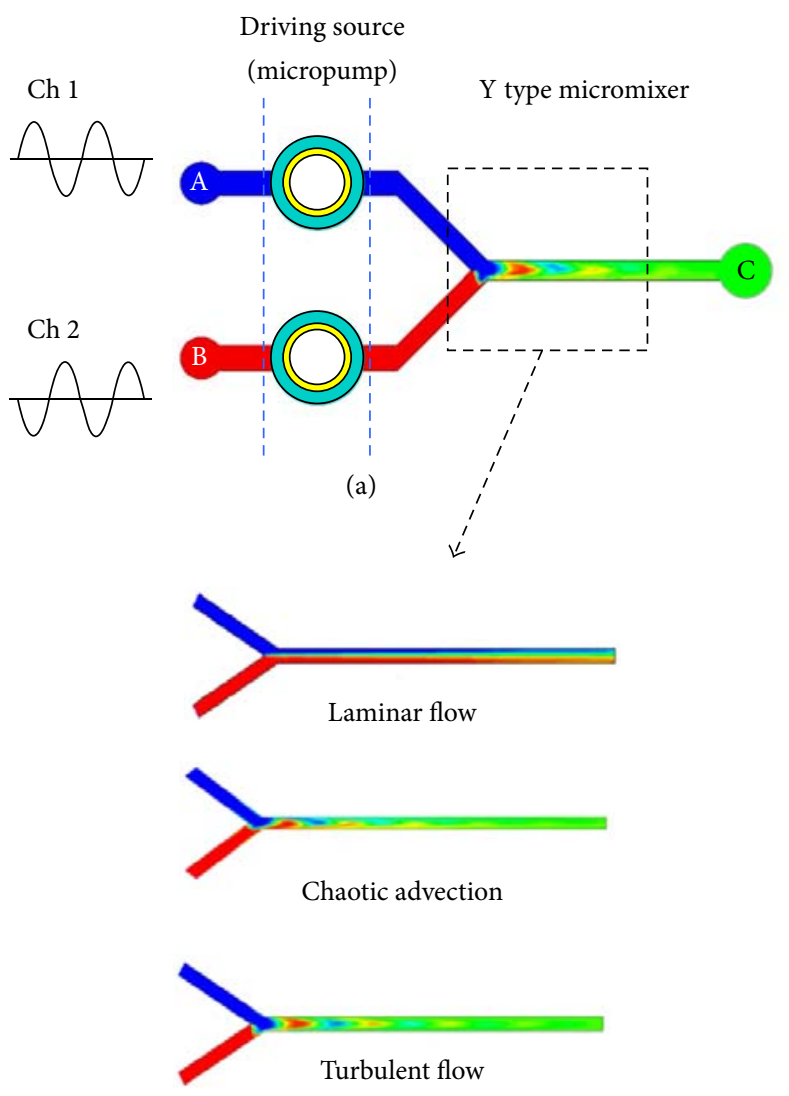

(b)

FIGURE 2: Schematic diagram of pulsed micromixing.

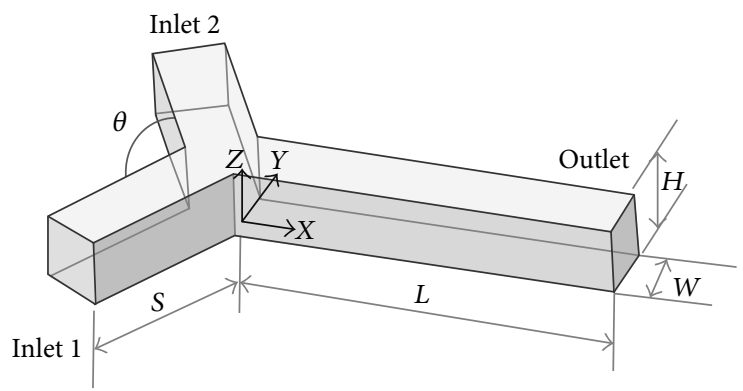

FIGURE 3: Simulation model of Y type micromixer.

different sizes of cross section, the four volume concentration distributions in the flow channel are analyzed by computational fluid dynamics (CFD) simulations, as shown in Figure 4. Analyzing the concentration distribution diagram of Figure 4(a), it can be clearly seen that, after one and a half cycles of pulsed mixing, the mixing effect of mixed solution is not obvious, and the boundary of two solution is still clear. The mixing effect has been improved a little in Figure 4(b), after one and a half pulsed mixing cycles. As for the Figure 4(c), the mixing is almost completed. In Figure 4(d), the field near the two sides of channel wall still has clear color boundary. Observing the concentration distribution diagram, it can be concluded that the mixing effect of Figure 4(c) is optimal. The simulation results indicate that when the entrance flow rate and working frequency are ascertained, there exist corresponding optimum values of the section size in order to obtain the best mixing effect. When the output volume of PZT micropump per pulse can be filled with the whole cross section, the microsolution presents a bullet type distribution in the flow channel. And the shorter the length of bullet is, the larger the contact area of two solutions is. Of course, the mixing effect will be better. While the output volume per pulse just can be filled with microchannel exactly, the mixing effect is the best. If it fails to fill the entire cross section, as shown in Figure 4(d), the mixing state belongs to the chaotic convection mode, and the mixing effect decreases compared with Figure 4(c). So when the flow rate and working frequency of micropumps are fixed, the size of cross section has an optimum value. Accordingly, the mixing degrees versus different section size and different section location are shown in Table 2. Based on the simulation results, comprehensively considering the fabrication process, structure, and saving the dosage of chemical reagent and other factors, the final section size after optimization is determined as $0.4 \mathrm{~mm} \times 0.4 \mathrm{~mm}$.

3.3.2. Analysis of Effects of Different Entrance Channel Angle on the Mixing Effect. The angle of entrance channel is another important structure parameter influencing the mixing effect. 


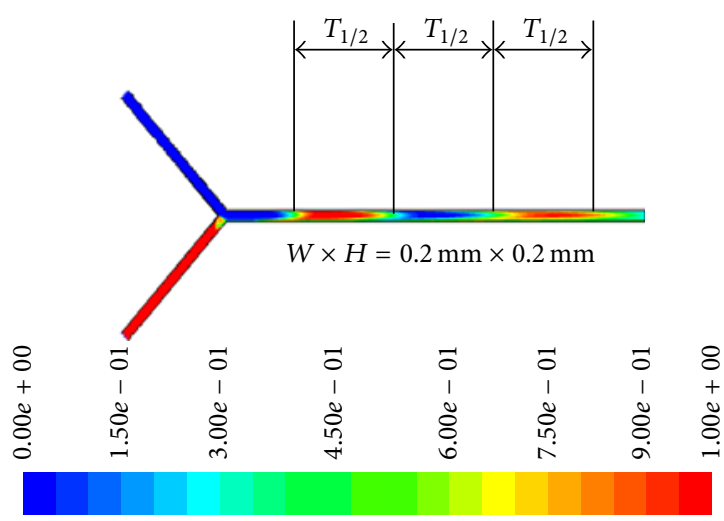

(a)

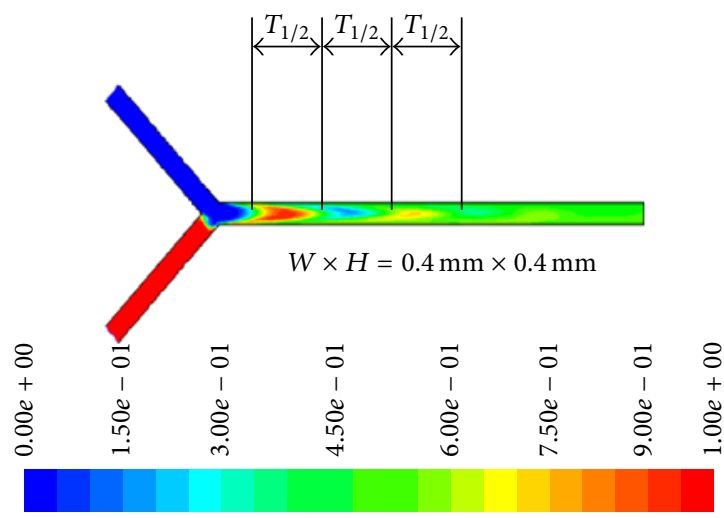

(c)

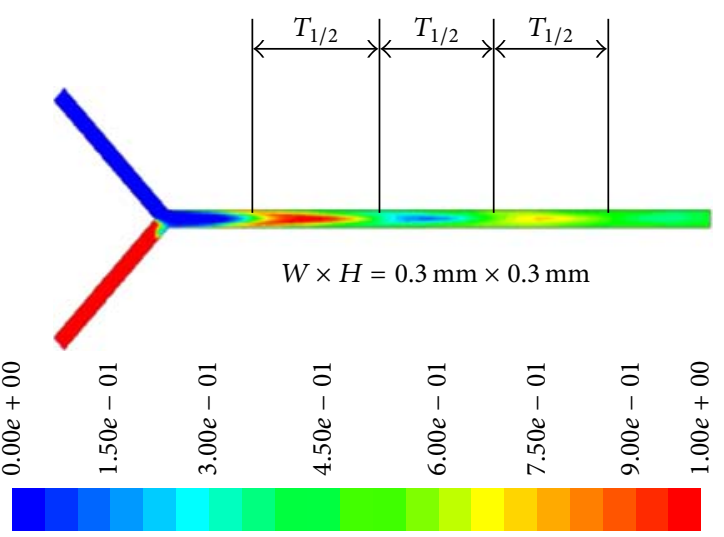

(b)

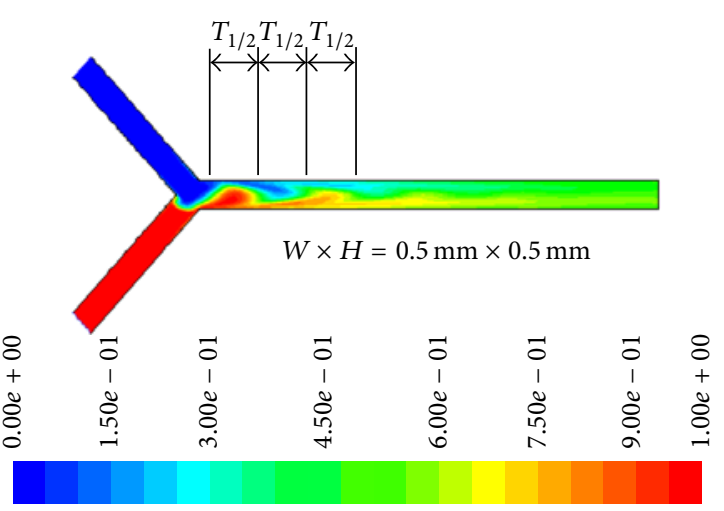

(d)

FIGURE 4: Concentration distributions of flow for different cross sections.

TABLE 1: Section size of microchannel and the corresponding velocity amplitude of entrance.

\begin{tabular}{lcccc}
\hline$W \times H(\mathrm{~mm})$ & $0.2 \times 0.2$ & $0.3 \times 0.3$ & $0.4 \times 0.4$ & $0.5 \times 0.5$ \\
\hline $\begin{array}{l}\text { Velocity amplitude } \\
V(\mathrm{~m} / \mathrm{s})\end{array}$ & 1.667 & 0.741 & 0.417 & 0.267 \\
\hline
\end{tabular}

TABle 2: Mixing degree of the fluid for different section size and location.

\begin{tabular}{lccc}
\hline$W \times H(\mathrm{~mm})$ & \multicolumn{3}{c}{ Mixing degree $\sigma$} \\
& $X=9$ & $X=10$ & $X=11$ \\
\hline $0.3 \times 0.3$ & 0.6341 & 0.6753 & 0.7892 \\
$0.4 \times 0.4$ & 0.8446 & 0.8761 & 0.9189 \\
$0.5 \times 0.5$ & 0.8537 & 0.8928 & 0.9187 \\
\hline
\end{tabular}

In this work, the effects of different entrance channel angle on the mixing effect are stimulated by CFD software, when section size is determined as $0.4 \mathrm{~mm} \times 0.4 \mathrm{~mm}$ with other conditions unchanged. The simulation results of volume concentration distribution in the mixing channel are shown in Figure 5. The volume concentration distributes in bullet shape, and the mixing effect by layer morphology is better than other angles due to more contact and diffusion areas in boundary. Table 3 lists the mixing degree value of mainstream
TABle 3: Mixing degree of the fluid phase 1 for different flow angles and locations.

\begin{tabular}{lcccccc}
\hline$X$ \\
$(\mathrm{~mm})$ & $\theta=30^{\circ}$ & $\theta=60^{\circ}$ & $\theta=90^{\circ}$ & $\theta=120^{\circ}$ & $\theta=150^{\circ}$ & $\theta=180^{\circ}$ \\
\hline$X=9$ & 0.8367 & 0.9104 & 0.8446 & 0.8118 & 0.7048 & 0.6610 \\
$X=10$ & 0.8557 & 0.9397 & 0.8761 & 0.8371 & 0.7449 & 0.6922 \\
$X=11$ & 0.8429 & 0.9084 & 0.9189 & 0.7898 & 0.6761 & 0.6261 \\
\hline
\end{tabular}

channel with different entrance channel angles and different section locations. The simulation results in Table 3 show that the mixing degree increases first and then decreases with the increase of angle between entrance channels. When the angle equals $60^{\circ}$, the maximum value of mixing degree is achieved, and three mixing degree values are all greater than 0.9 . Therefore, $60^{\circ}$ is finally selected as the optimal angle of entrance channels.

3.4. Optimization of the Working Parameter. For the pulsed micromixing system, the working frequency and entrance flow rate are the most important working parameters which directly affect the mixing effect. Since the testing system adopts the mature driving technology of PZT micropump, the optimization of working parameters focuses on the research of working frequency. The specific frequency in the range 


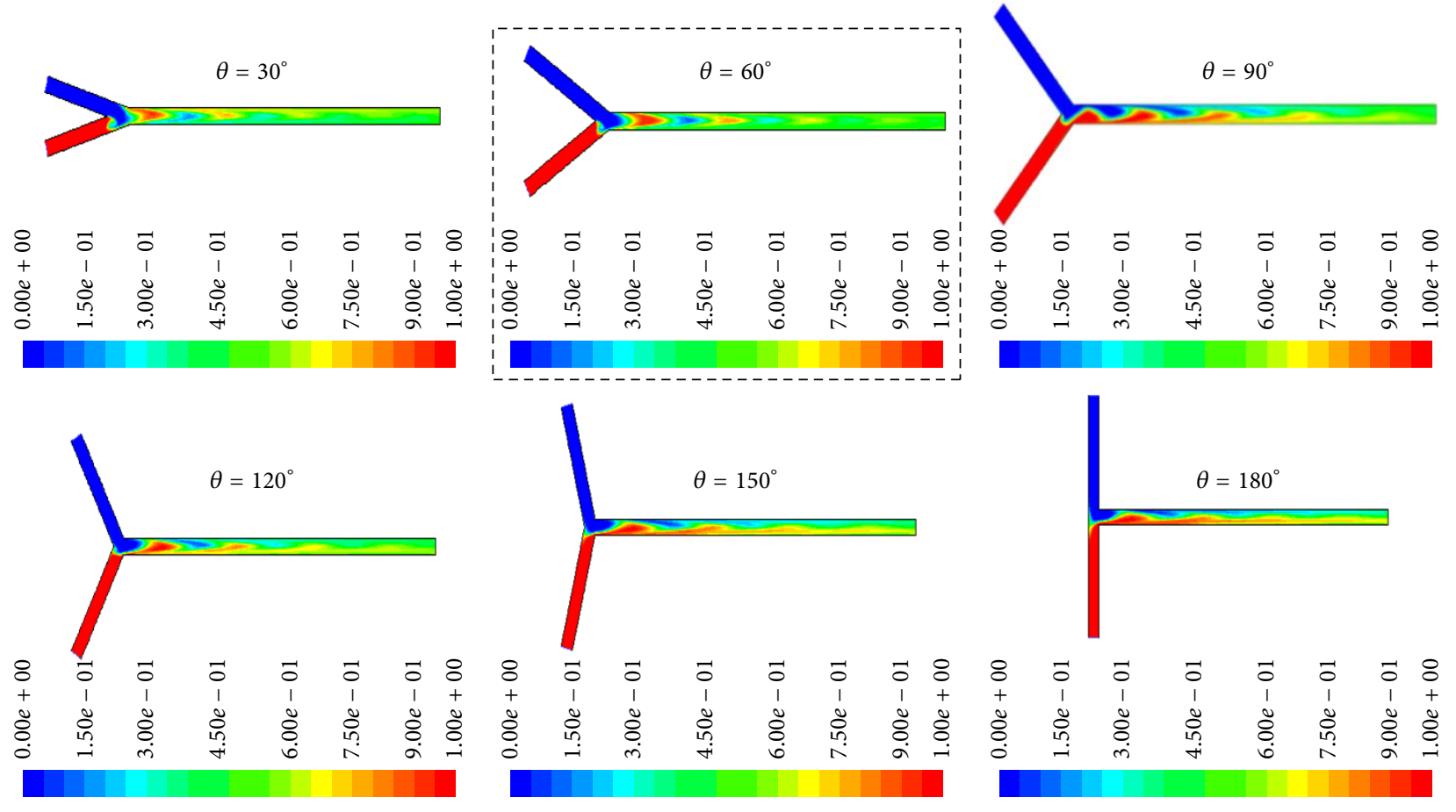

FIGURE 5: Concentration distributions of flow for different flow angle.

of $50 \sim 350 \mathrm{~Hz}$ is studied and analyzed for its influence on the mixing effect, assuming that the entrance flow rate is $4 \mathrm{~mL} / \mathrm{min}$. The simulation results of CFD and the relationship curves between the mixing degree and working frequency are shown in Figure 6. Analyzing the distribution graphs and curves in Figure 6, it can be concluded that when the flow rate remains constant $(4 \mathrm{~mL} / \mathrm{min})$, the mixing degree increases first and then decreases with the increase of working frequency. When the frequency is $300 \mathrm{~Hz}$, the mixing degree reaches the maximum value at 0.97 , which realizes a nearly complete mixture. Under this condition, the mixing effect is optimal.

\section{Experiment}

4.1. Reagents. $\mathrm{HAuCl}_{4} \cdot 4 \mathrm{H}_{2} \mathrm{O}$ (precursor), $\mathrm{Na}_{3} \mathrm{C}_{6} \mathrm{H}_{5} \mathrm{O}_{7}$ (reducing agent), and CTAB (cationic surface active agent) were purchased from Sinopharm Chemical Reagent Corporation (Shanghai, China). The other reagents were of the highest grades available commercially. The water used in the experiments was of Milli-Q grade.

4.2. Apparatus. UV-Vis absorption spectrophotometer (UV2501 PC, SHIMADZU, Japan) was used to record the characteristic absorption spectrum of gold colloid. The size and morphology of synthesized AuNPs were characterized by high resolution transmission electron microscopy (TEM TECNAI-12, Philips, Holland). The PZT micropump was fabricated using mature MEMS process in lab [35]. The output flow rate of micropump can be adjusted in the range of $0 \sim 6 \mathrm{~mL} / \mathrm{min}$, and the working frequency of micropump can be adjusted in the range of $10 \sim 400 \mathrm{~Hz}$. The fabrication of $\mathrm{Y}$ type micromixer used a computer-numerically controlled (CNC) machining process and a polydimethylsiloxane (PDMS) replication process [4]. The mixing channel and reaction channel were packaged using PDMS and glass. Also, the design and manufacture of micromixer all adopted the optimized structural parameters above. The constant temperature heating platform (BHW-05A shanghai, China) was used to provide the stable reaction temperature. In addition, precision electronic balance and power supply controller were used in experiment test.

Figure 7 shows the scheme of the experimental setup, the prototype photo of PZT micropumps, and testing photo of controlled synthesis of gold nanoparticles using pulsed mixing microfluidic system. The detailed experimental procedure is as follows. First, all glassware used was clean in freshly prepared aqua regia solution $\left(\mathrm{HCI}: \mathrm{HNO}_{3}, 3: 1\right)$ and rinsed with deionized water three times before used. Before the synthesis of AuNPs, the chambers of PZT micropump and the channels of micromixer were cleaned by deionized water. The gold precursor, reducing agent, and surfactant were, respectively, dissolved in deionized water to prepare the reaction reagents of the corresponding concentration [36], which include $100 \mathrm{~mL} \mathrm{HAuCl}_{4}$ of $1 \mathrm{mM}, 100 \mathrm{~mL} \mathrm{Na}_{3} \mathrm{C}_{6} \mathrm{H}_{5} \mathrm{O}_{7}$ of $1.5 \mathrm{mM}$, and $100 \mathrm{~mL}$ CTAB of $200 \mathrm{mM}$. The temperature control platform was adjusted to keep the constant temperature of $100^{\circ} \mathrm{C}$. With adjustments of the working voltage, the flow rate of PZT micropumps was fixed at $4 \mathrm{~mL} / \mathrm{min}$. To achieve the crossing pulsing micromixing of two reaction reagents, the phase of input signal of PZT micropumps was regulated to maintain $180^{\circ}$. After turning on the power of PZT micropumps, the gold precursor $\left(\mathrm{HAuCl}_{4}\right)$ and the reducing 

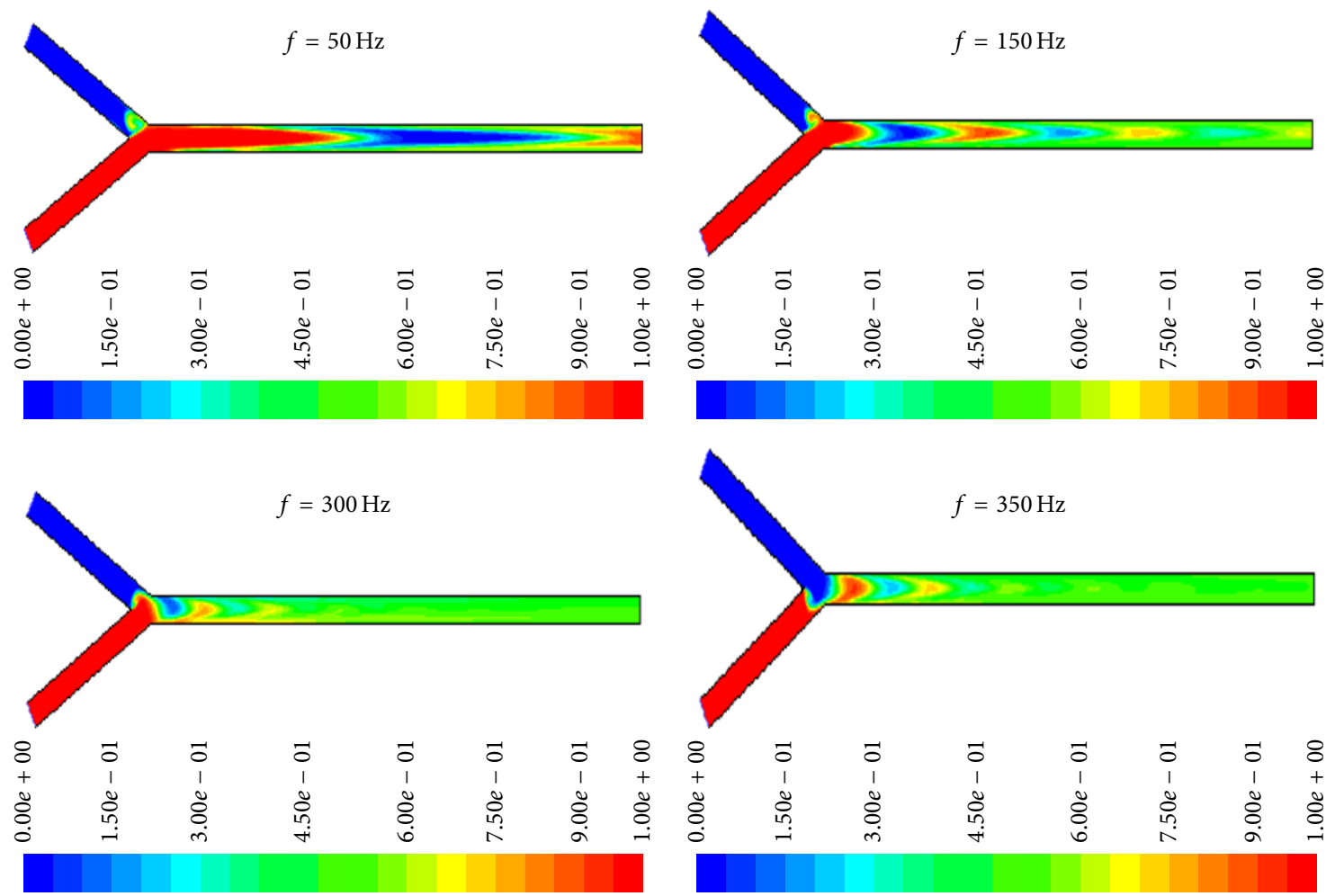

(a)

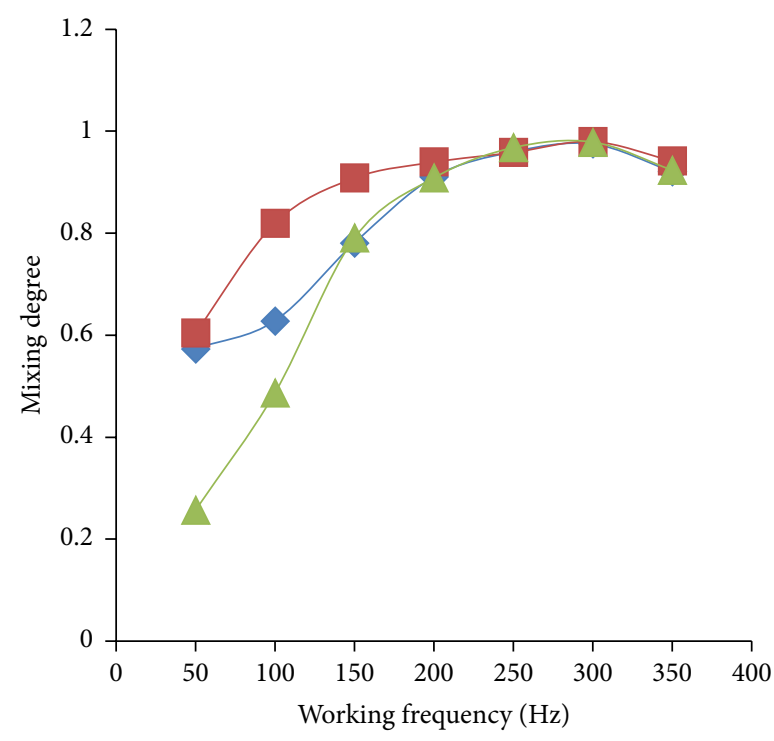

- The mixing degree of $X=9$ section in different working frequency

- The mixing degree of $X=10$ section in different working frequency

- The mixing degree of $X=11$ section in different working frequency

(b)

FIGURE 6: Concentration distributions of flow for different working frequencies and relationship curves between working frequency and mixing degree in different sections of microchannel. 


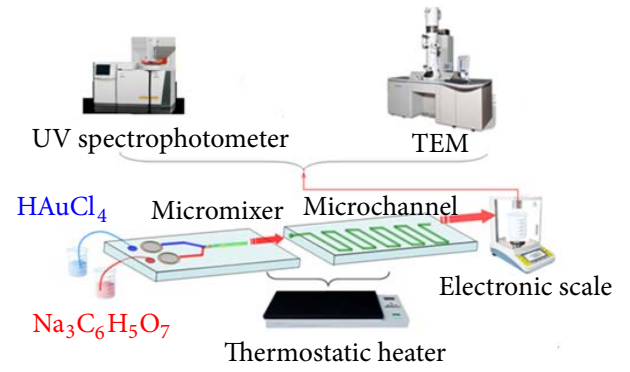

(a)

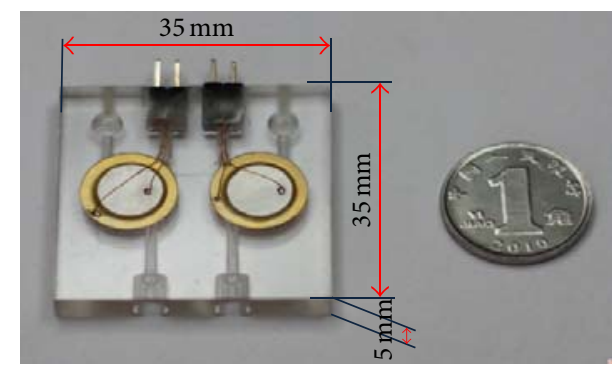

(b)

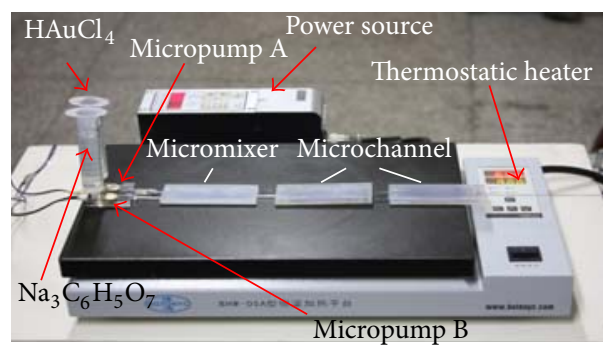

(c)

Figure 7: (a) Experimental scheme; (b) the prototype photo of PZT micropumps; (c) testing photo of controlled synthesis of gold nanoparticles using pulsed mixing microfluidic system.

agent $\left(\mathrm{Na}_{3} \mathrm{C}_{6} \mathrm{H}_{5} \mathrm{O}_{7}\right)$ were pumped into the micromixer and growth channel by the crossing pulsing state. At the outlet of microchannel, the solution which experienced the "pulsed mixing" and passed through the "growth flow channel" was collected. At the same time, in order to avoid the agglomeration of AuNPs, the corresponding amount of CTAB was also added to the collected samples one time in proportion $\left(n_{\mathrm{CTAB}} / n_{\mathrm{HAuCl} 4}=200: 1\right)$. After about ten minutes, the synthesized AuNPs reached stable state, and then the size and morphology of AuNPs can be characterized using the relevant testing apparatus. Note that the experiments were conducted in the frequency of 100,200 , and $300 \mathrm{~Hz}$, respectively.

4.3. Measurement. To monitor the formation of gold nanostructures, UV-Vis spectroscopy was used to characterize the resulting samples in real-time by UV-Vis absorption spectrophotometer. The size and morphology of AuNPs synthesized based on different frequencies were characterized by transmission electron microscope (TEM). The size distribution of synthesized AuNPs was calculated and analyzed by measuring 300 nanoparticles (six groups) in each sample based on TEM micrographs with ImageJ software.

\section{Results and Discussion}

5.1. Test Results. Table 4 summarizes the values characterized for AuNPs synthesized at different frequency. (where $\lambda_{m}$ is the wavelength of absorption maximum, $\alpha_{m}$ is the absorption maximum, PWHM is peak width at half maximum, $\overline{n_{m}}$ is an average diameter of nanoparticles, and $\sigma$ is the standard deviation of particles size).
TABLE 4: Three groups of experimental results.

\begin{tabular}{lcccccc}
\hline Number & $f / \mathrm{Hz}$ & $\lambda_{m} / \mathrm{nm}$ & $\alpha_{m}$ & PWHM $/ \mathrm{nm}$ & $\overline{n_{m}} / \mathrm{nm}$ & $\sigma / \mathrm{nm}$ \\
\hline 1 & 100 & 534 & 0.852 & 96 & 25.8 & 6.08 \\
2 & 200 & 532 & 1.0191 & 90 & 28.6 & 5.47 \\
3 & 300 & 524 & 0.6804 & 84 & 21.6 & 4.83 \\
\hline
\end{tabular}

5.2. Analysis of Effect of Pulsing Frequency. Figure 8 shows the UV-Vis spectra of three kinds of AuNPs synthesized under different mixing frequencies, when the entrance flow rate is fixed at $4 \mathrm{~mL} / \mathrm{min}$. And the corresponding frequencies of the samples (numbers 1, 2, and 3) are 100, 200, and $300 \mathrm{~Hz}$, respectively. As shown in Figure 8, with the increase of pulsing mixing frequency, the absorbance of sample first increases and then decreases. Accordingly, the absorbance maximum of samples rises from 0.852 to 1.091 and then drops to 0.6804 . While the absorbance maximum reflects the concentration of particles size, which indicates that the concentration of AuNPs of sample number 2 is the largest, the concentration of AuNPs of sample number 3 is the minimum. As shown in Table 4 and Figure 8, it is also observed that, with the increase of mixing frequency, a continuous blueshift of the wavelength of absorption maximum occurs. To be more specific, the corresponding wavelength shifts from $534 \mathrm{~nm}$ to $532 \mathrm{~nm}$ and then blue-shifted to $524 \mathrm{~nm}$. Note that samples (numbers 1, 2, and 3) of synthesized AuNPs showed the typical and unique optical characteristics of gold colloid, and surface plasmon resonance (SPR) by AuNPs was observed at 500 550 nm [37, 38], which further confirms the synthesis of AuNPs. Analyzing the UV-Vis spectra of prepared AuNPs in detail, we can know that the PWHM 


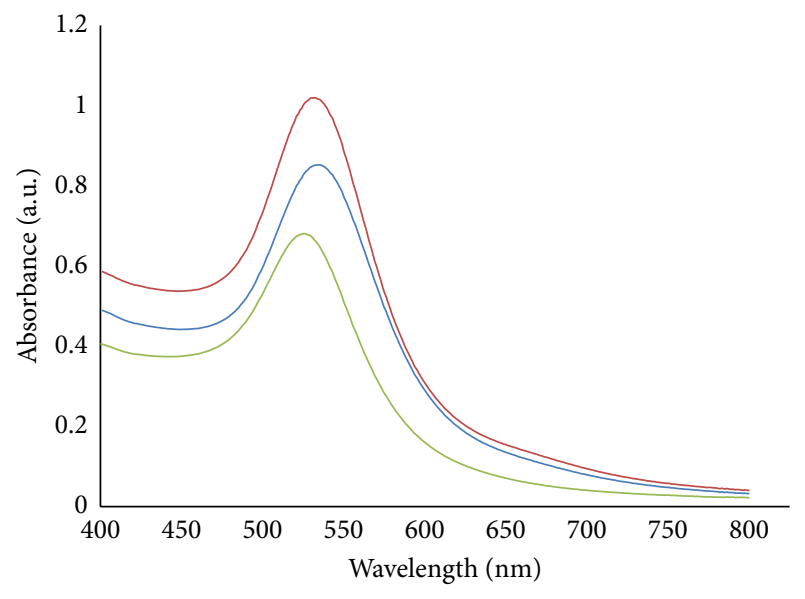

Number $1-100 \mathrm{~Hz}$

Number $2-200 \mathrm{~Hz}$

Number $3-300 \mathrm{~Hz}$

FIGURE 8: UV-Vis absorption spectra of gold colloids at different frequencies.

values of three samples are $96 \mathrm{~nm}$ (number 1), $90 \mathrm{~nm}$ (number 2), and $84 \mathrm{~nm}$ (number 3), respectively. Actually, PWHM value is the peak width at half of absorption maximum, which can characteristic the monodispersity of AuNPs. The lower the PWHM value is, the narrower the size distribution of nanoparticles is [39]. Therefore, it can be inferred that particle diameter deviation of sample number 3 is the lowest, and number 1 is the largest. The inferences are confirmed further through the TEM observations.

Figure 9 shows the TEM images and the size distribution histogram of synthesized AuNPs, photos of gold of their corresponding gold colloids. As the photos of Figure 9 show, it can be clearly observed that the color of sample number 1 is pink, number is wine red, and number 3 is light pink, which is in good agreements with the analysis results of UV-Vis absorption spectra presented above. The higher the intensity of absorbance is, the larger the concentration of nanoparticles is. Analyzing the TEM images, we can find that most of AuNPs synthesized by the mixing frequency of $100 \mathrm{~Hz}$ are of irregular shape, and the particle monodispersity is poorer. The mean particle size and standard deviation of sample number 1 are $25.8 \pm 6.8 \mathrm{~nm}$. When the mixing frequency was set at $200 \mathrm{~Hz}$, the obtained nanoparticles showed good monodispersity, but the morphology of AuNPs still does not reach the expected level. The spherical or quasispherical particles account for a higher ratio, and the mean particle size and standard deviation are $28.6 \pm 5.47 \mathrm{~nm}$. With the frequency increasing to $300 \mathrm{~Hz}$, the resulting nanoparticles show better sphericity, most of which are spherical and, meanwhile, have a good monodispersion. The corresponding mean particle size and standard deviation are $21.6 \pm 4.83 \mathrm{~nm}$, which indicates that the AuNPs with smaller diameter and narrower size distribution are prepared successfully. The experimental results obtained above indicate that AuNPs synthesized under the mixing frequency of $300 \mathrm{~Hz}$ have the optimal size uniformity, morphology, and monodispersity, which are in good accordance with the results of previous simulation optimization. Also, the simulation analysis above showed that mixing in case of $300 \mathrm{~Hz}$ had the highest mixing degree. That is to say, the mixing effect under this mixing condition was the best. Exploring the deeper causes of test results, we consider that when the entrance flow rate keeps a certain value, different structure parameters (such as the section size and angle) and different working parameters will lead to kinds of different mixing effects. In the case all parameters are best matched, micropulsing mixing system will achieve the best mixing effects. In this condition, the two solutions layer at the $\mathrm{Y}$ type mixing channel became thinner, and the contact areas and opportunities of reaction reagents increased. Then, more fully mixing and reaction will be achieved. As Figure 1 shows, perfect mixing and reaction will directly shorten the nuclear reaction time (stage II: nucleation). At the same time, the total quantity of AuNPs will be ascertained quickly and then reaction entered the next stage (stage III: growth of crystal nucleus). Since two solutions have realize full mixing before entering the growth channel of nanoparticles, the growth environment of nanoparticles is equal, which ensures the final synthesis of nanoparticles with more uniform size and morphology.

\section{Conclusions}

Continuous synthesis of AuNPs using pulsed mixing microfluidic system has been carried out. The structure and working parameters for $\mathrm{Y}$ type micromixer have also been optimized, which includes the optimal analysis of section size, entrance channel angle, and pulse frequency on the mixing effect. According to the optimized structural parameters and working parameters, a corresponding pulsed mixing system prototype has been manufactured, and AuNPs with relatively uniform size and morphology have been successfully synthesized. Through the research above, the following conclusions are drawn:

(1) The pulsed micromixing system actuated PZT micropumps are competent to implement continuous, high-efficiency, controlled synthesis of AuNPs, and the prepared nanoparticles have relatively uniform size, morphology, and good monodispersity.

(2) The structure parameters (e.g., the section size and entrance angle) and working parameters (e.g., frequency, voltage, and phase of electrical signal) are significant important factors affecting the particles size and morphology by using pulsed micromixing system for synthesizing AuNPs. Optimizing the structure and operating parameters are an important technological link in the design and fabrication of pulsing micromixing system. The higher the mixing degree of mixing system is, the better the mixing and reaction effect is, and the particle size of nanoparticles prepared is smaller; size distribution of nanoparticles is also narrower.

The preparation of AuNPs in liquid phase is a complex chemical reaction process. Many effect facts such as the concentration of the reactants, $\mathrm{pH}$ value, temperature, UV 

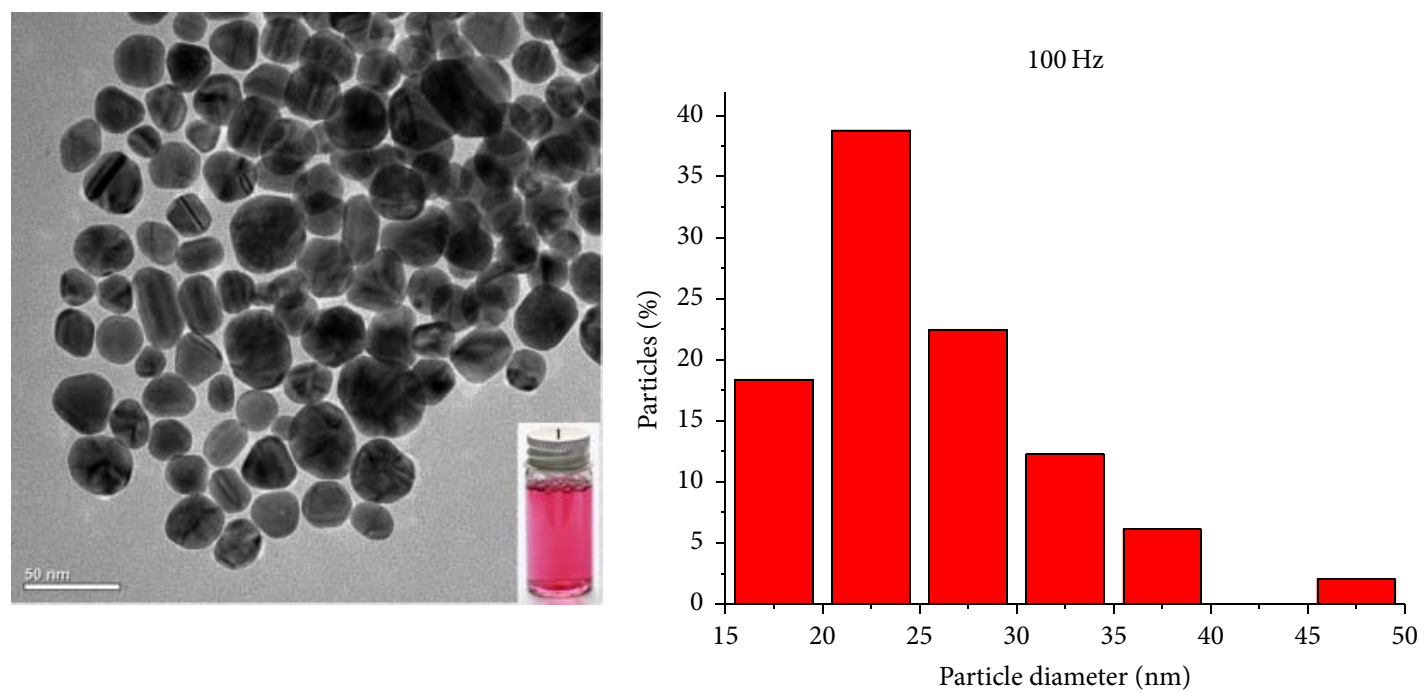

(a)
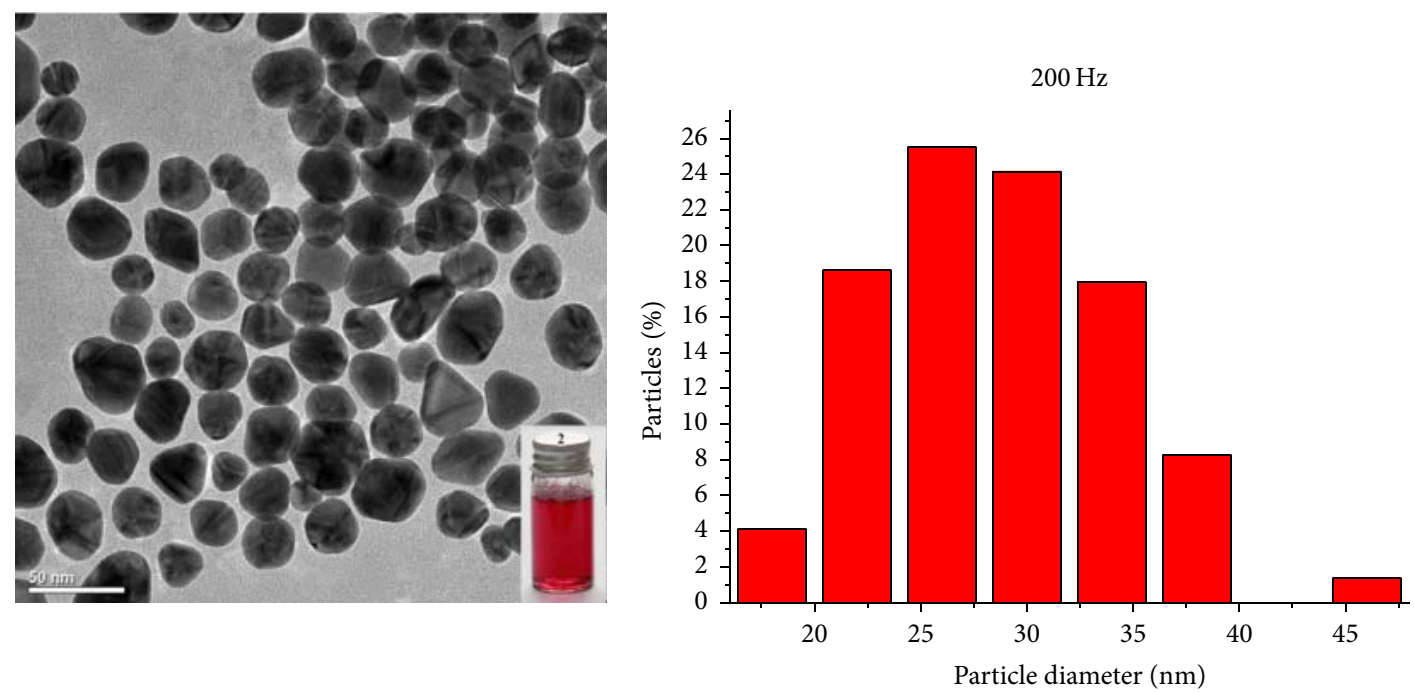

(b)
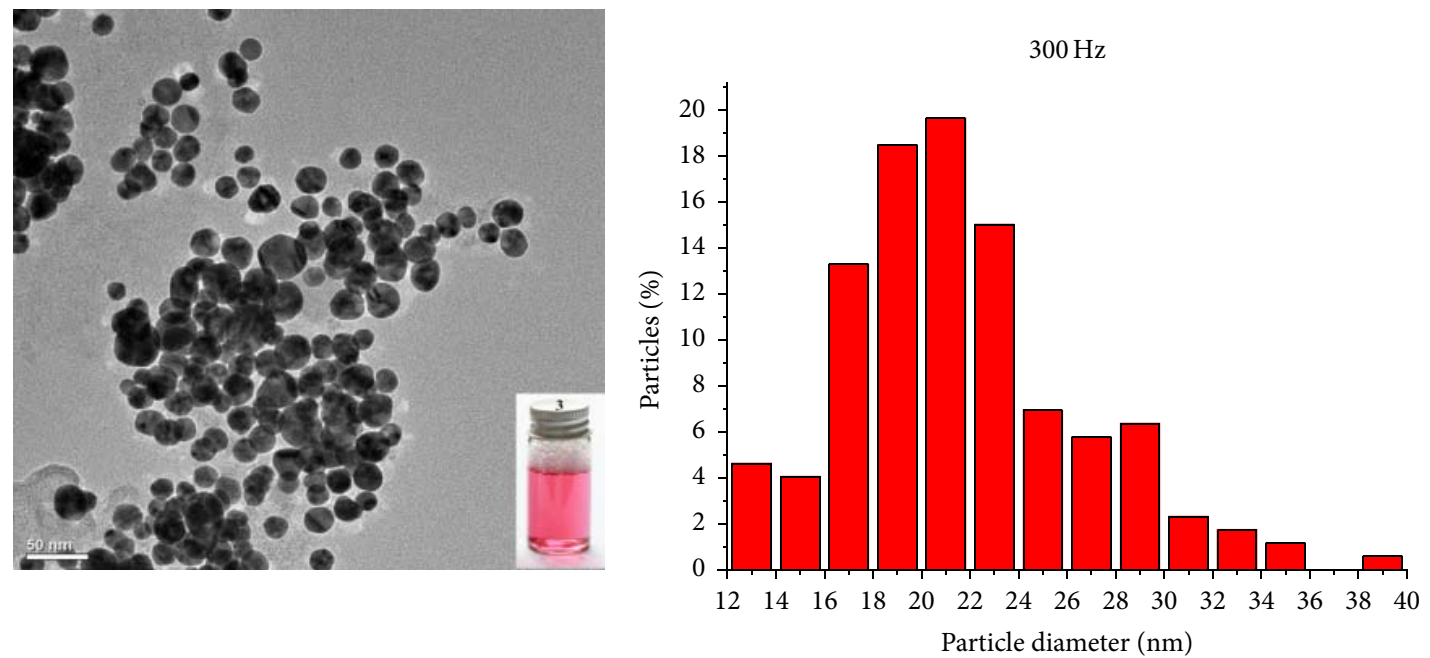

(c)

FIGURE 9: TEM micrographs and histograms of gold nanoparticles at different frequencies ((a)-(c) in $f=100 \mathrm{~Hz}, f=200 \mathrm{~Hz}$, and $f=300 \mathrm{~Hz}$ conditions). 
light, and ultrasonic vibration will affect the particle size and morphology of nanoparticles. In the future, the pulsed micromixing system will be further optimized, and the more comprehensive, systematic controlled preparation of nanoparticles will be carried out, on the basis of considering many influence factors.

\section{Conflict of Interests}

The authors declare that there is no conflict of interests regarding the publication of this paper.

\section{Acknowledgments}

This research is supported and funded by the National Natural Science Foundation Projects (no. 51375207) from Ministry of Science and Technology of the People's Republic of China. Thanks are due to the chain drive research institution of Jilin University for the energetic support and help in the aspect of microdevices fabrication.

\section{References}

[1] U. Shedbalkar, R. Singh, S. Wadhwani, S. Gaidhani, and B. A. Chopade, "Microbial synthesis of gold nanoparticles: current status and future prospects," Advances in Colloid and Interface Science, vol. 209, pp. 40-48, 2014.

[2] S. Eustis and M. A. El-Sayed, "Why gold nanoparticles are more precious than pretty gold: noble metal surface plasmon resonance and its enhancement of the radiative and nonradiative properties of nanocrystals of different shapes," Chemical Society Reviews, vol. 35, no. 3, pp. 209-217, 2006.

[3] L. Zou, W. Qi, R. Huang, R. Su, M. Wang, and Z. He, "Green synthesis of a gold nanoparticle-nanocluster composite nanostructures using trypsin as linking and reducing agents," ACS Sustainable Chemistry and Engineering, vol. 1, no. 11, pp. 1398-1404, 2013.

[4] S.-Y. Yang, F.-Y. Cheng, C.-S. Yeh, and G.-B. Lee, "Sizecontrolled synthesis of gold nanoparticles using a micro-mixing system," Microfluidics and Nanofluidics, vol. 8, no. 3, pp. 303-311, 2010.

[5] Y. Xiong, B. J. Wiley, and Y. Xia, "Nanocrystals with unconventional shapes-a class of promising catalysts," Angewandte Chemie-International Edition, vol. 46, no. 38, pp. 7157-7159, 2007.

[6] A. Corma and H. Garcia, "Supported gold nanoparticles as catalysts for organic reactions," Chemical Society Reviews, vol. 37, no. 9, pp. 2096-2126, 2008.

[7] C. V. Navin, K. S. Krishna, C. S. Theegala, and C. S. S. R. Kumar, "Lab-on-a-chip devices for gold nanoparticle synthesis and their role as a catalyst support for continuous flow catalysis," Nanotechnology Reviews, vol. 3, no. 1, pp. 39-63, 2014.

[8] T. A. Taton, G. Lu, and C. A. Mirkin, "Two-color labeling of oligonucleotide arrays via size-selective scattering of nanoparticle probes," Journal of the American Chemical Society, vol. 123, no. 21, pp. 5164-5165, 2001.

[9] M.-C. Daniel and D. Astruc, "Gold nanoparticles: assembly, supramolecular chemistry, quantum-size-related properties, and applications toward biology, catalysis, and nanotechnology," Chemical Reviews, vol. 104, no. 1, pp. 293-346, 2004.
[10] J. N. Anker, W. P. Hall, O. Lyandres, N. C. Shah, J. Zhao, and R. P. Van Duyne, "Biosensing with plasmonic nanosensors," Nature Materials, vol. 7, no. 6, pp. 442-453, 2008.

[11] A. J. Mieszawska, W. J. M. Mulder, Z. A. Fayad, and D. P. Cormode, "Multifunctional gold nanoparticles for diagnosis and therapy of disease," Molecular Pharmaceutics, vol. 10, no. 3, pp. 831-847, 2013.

[12] X. Huang, I. H. El-Sayed, W. Qian, and M. A. El-Sayed, "Cancer cell imaging and photothermal therapy in the nearinfrared region by using gold nanorods," Journal of the American Chemical Society, vol. 128, no. 6, pp. 2115-2120, 2006.

[13] E. Boisselier and D. Astruc, "Gold nanoparticles in nanomedicine: preparations, imaging, diagnostics, therapies and toxicity," Chemical Society Reviews, vol. 38, no. 6, pp. 1759-1782, 2009.

[14] C. M. Cobley, J. Chen, E. C. Cho, L. V. Wang, and Y. Xia, "Gold nanostructures: a class of multifunctional materials for biomedical applications," Chemical Society Reviews, vol. 40, no. 1, pp. 44-56, 2011.

[15] E. C. Dreaden, A. M. Alkilany, X. Huang, C. J. Murphy, and M. A. El-Sayed, "The golden age: gold nanoparticles for biomedicine," Chemical Society Reviews, vol. 41, no. 7, pp. 2740 2779, 2012.

[16] J. Panyam and V. Labhasetwar, "Biodegradable nanoparticles for drug and gene delivery to cells and tissue," Advanced Drug Delivery Reviews, vol. 55, no. 3, pp. 329-347, 2003.

[17] M. Liong, J. Lu, M. Kovochich et al., "Multifunctional inorganic nanoparticles for imaging, targeting, and drug delivery," ACS Nano, vol. 2, no. 5, pp. 889-896, 2008.

[18] T. L. Doane and C. Burda, "The unique role of nanoparticles in nanomedicine: imaging, drug delivery and therapy," Chemical Society Reviews, vol. 41, no. 7, pp. 2885-2911, 2012.

[19] N. Nath and A. Chilkoti, "A colorimetric gold nanoparticle sensor to interrogate biomolecular interactions in real time on a surface," Analytical Chemistry, vol. 74, no. 3, pp. 504-509, 2002.

[20] K. Shibamoto, K. Katayama, M. Fujinami, and T. Sawada, "Fundamental processes of surface enhanced Raman scattering detected with transient reflecting grating spectroscopy," Review of Scientific Instruments, vol. 74, no. 1, pp. 910-912, 2003.

[21] Á. I. López-Lorente, M. Valcárcel, and B. Mizaikoff, "Continuous flow synthesis and characterization of tailor-made bare gold nanoparticles for use in SERS," Microchimica Acta, vol. 181, no. 9-10, pp. 1101-1108, 2014.

[22] K. Sugano, Y. Uchida, O. Ichihashi, H. Yamada, T. Tsuchiya, and O. Tabata, "Mixing speed-controlled gold nanoparticle synthesis with pulsed mixing microfluidic system," Microfluidics and Nanofluidics, vol. 9, no. 6, pp. 1165-1174, 2010.

[23] D. V. R. Kumar, A. A. Kulkarni, and B. L. V. Prasad, "Microfluidic platform for continuous flow synthesis of triangular gold nanoplates," Colloids and Surfaces A: Physicochemical and Engineering Aspects, vol. 443, pp. 149-155, 2014.

[24] S. P. Chandran, M. Chaudhary, R. Pasricha, A. Ahmad, and M. Sastry, "Synthesis of gold nanotriangles and silver nanoparticles using aloevera plant extract," Biotechnology Progress, vol. 22, no. 2, pp. 577-583, 2006.

[25] J. A. Dahl, B. L. S. Maddux, and J. E. Hutchison, “Toward greener nanosynthesis," Chemical Reviews, vol. 107, no. 6, pp. 2228-2269, 2007.

[26] L. Minati, F. Benetti, A. Chiappini, and G. Speranza, "Onestep synthesis of star-shaped gold nanoparticles," Colloids and Surfaces A: Physicochemical and Engineering Aspects, vol. 441, pp. 623-628, 2014. 
[27] H. C. Lee and C. H. Lin, "Liquid-liquid phase separator for synthesizing gold nanoparticles in toluene," in Proceedings of the 9th Annual IEEE International Conference on Nano/Micro Engineered and Molecular Systems (NEMS '14), Honolulu, Hawaii, USA, April 2014.

[28] C.-X. Zhao, L. He, S. Z. Qiao, and A. P. J. Middelberg, "Nanoparticle synthesis in microreactors," Chemical Engineering Science, vol. 66, no. 7, pp. 1463-1479, 2011.

[29] G. A. Patil, M. L. Bari, B. A. Bhanvase, V. Ganvir, S. Mishra, and S. H. Sonawane, "Continuous synthesis of functional silver nanoparticles using microreactor: effect of surfactant and process parameters," Chemical Engineering and Processing: Process Intensification, vol. 62, pp. 69-77, 2012.

[30] M. Faryadi, M. Rahimi, S. Safari, and N. Moradi, "Effect of high frequency ultrasound on micromixing efficiency in microchannels," Chemical Engineering and Processing: Process Intensification, vol. 77, pp. 13-21, 2014.

[31] J. S. Zhang, K. Wang, Y. C. Lu, and G. S. Luo, "Characterization and modeling of micromixing performance in micropore dispersion reactors," Chemical Engineering and Processing: Process Intensification, vol. 49, no. 7, pp. 740-747, 2010.

[32] F. Parvizian, M. Rahimi, and M. Faryadi, "Macro- and micromixing in a novel sonochemical reactor using high frequency ultrasound," Chemical Engineering and Processing, vol. 50, no. 8, pp. 732-740, 2011.

[33] M. Jarrahi, C. Castelain, and H. Peerhossaini, "Mixing enhancement by pulsating chaotic advection," Chemical Engineering and Processing, vol. 74, pp. 1-13, 2014.

[34] S. M. Shin, I. S. Kang, and Y.-K. Cho, "Mixing enhancement by using electrokinetic instability under time-periodic electric field," Journal of Micromechanics and Microengineering, vol. 15, no. 3, pp. 455-462, 2005.

[35] G. J. Liu, Z. G. Yang, J. F. Liu et al., "A low cost, high performance insulin delivery system based on PZT actuation," Microsystem Technologies, vol. 20, no. 12, pp. 2287-2294, 2014.

[36] B. Mao, B. Liu, and Y. F. Wang, "Preparation of Au colloid of small size in aqueous solution," Rare Metal Materials and Engineering, vol. 38, no. 3, pp. 515-518, 2009.

[37] K. Aslan and V. H. Pérez-Luna, "Surface modification of colloidal gold by chemisorption of alkanethiols in the presence of a nonionic surfactant," Langmuir, vol. 18, no. 16, pp. 6059$6065,2002$.

[38] N. R. Jana, L. Gearheart, and C. J. Murphy, "Seeding growth for size control of 5-40 nm diameter gold nanoparticles," Langmuir, vol. 17, no. 22, pp. 6782-6786, 2001.

[39] K. R. Brown, D. G. Walter, and M. J. Natan, "Seeding of colloidal $\mathrm{Au}$ nanoparticle solutions. 2. Improved control of particle size and shape," Chemistry of Materials, vol. 12, no. 2, pp. 306-313, 2000 . 

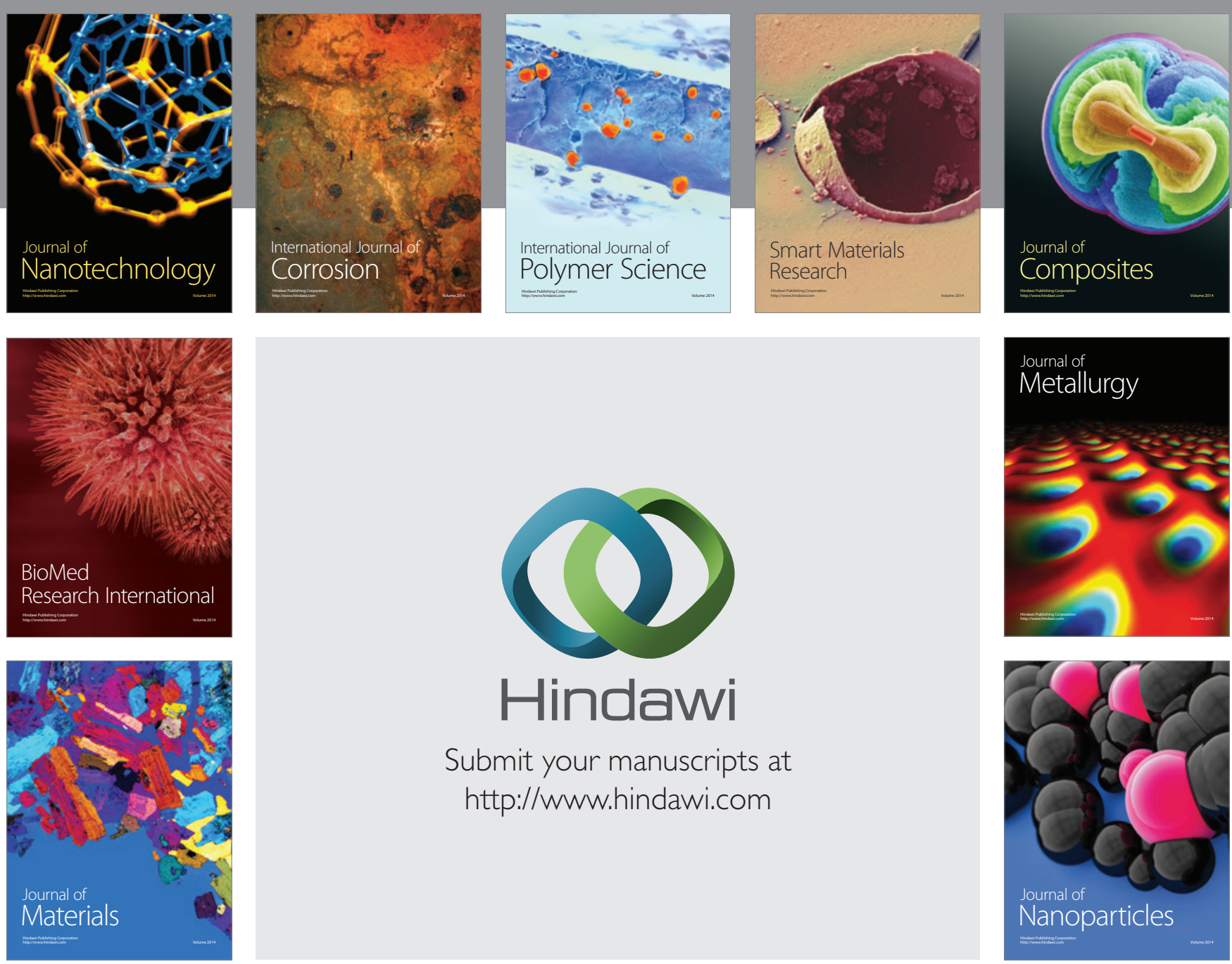

Submit your manuscripts at http://www.hindawi.com
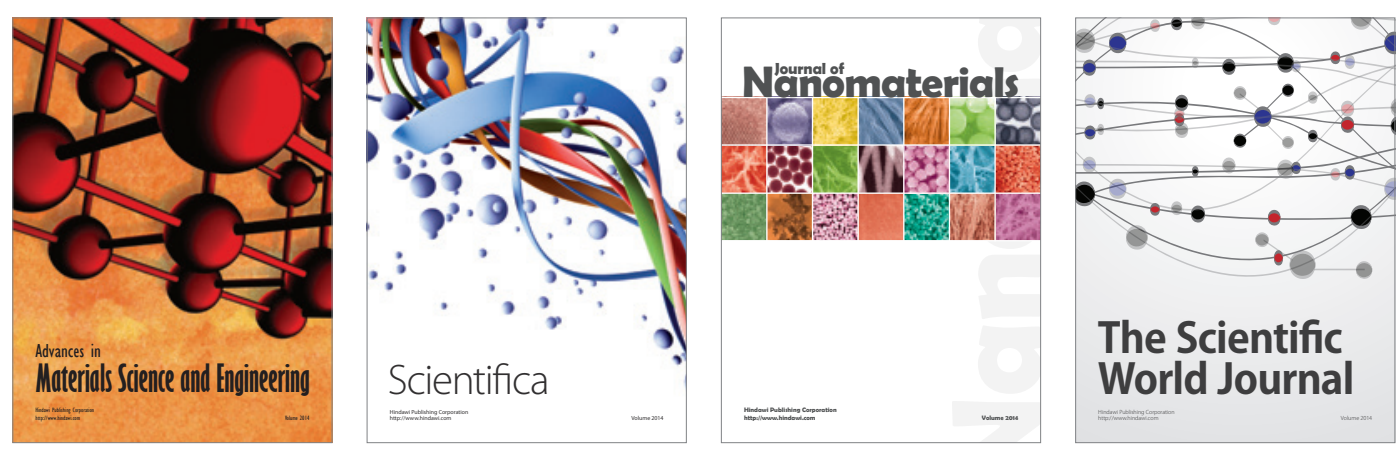

\section{The Scientific World Journal}
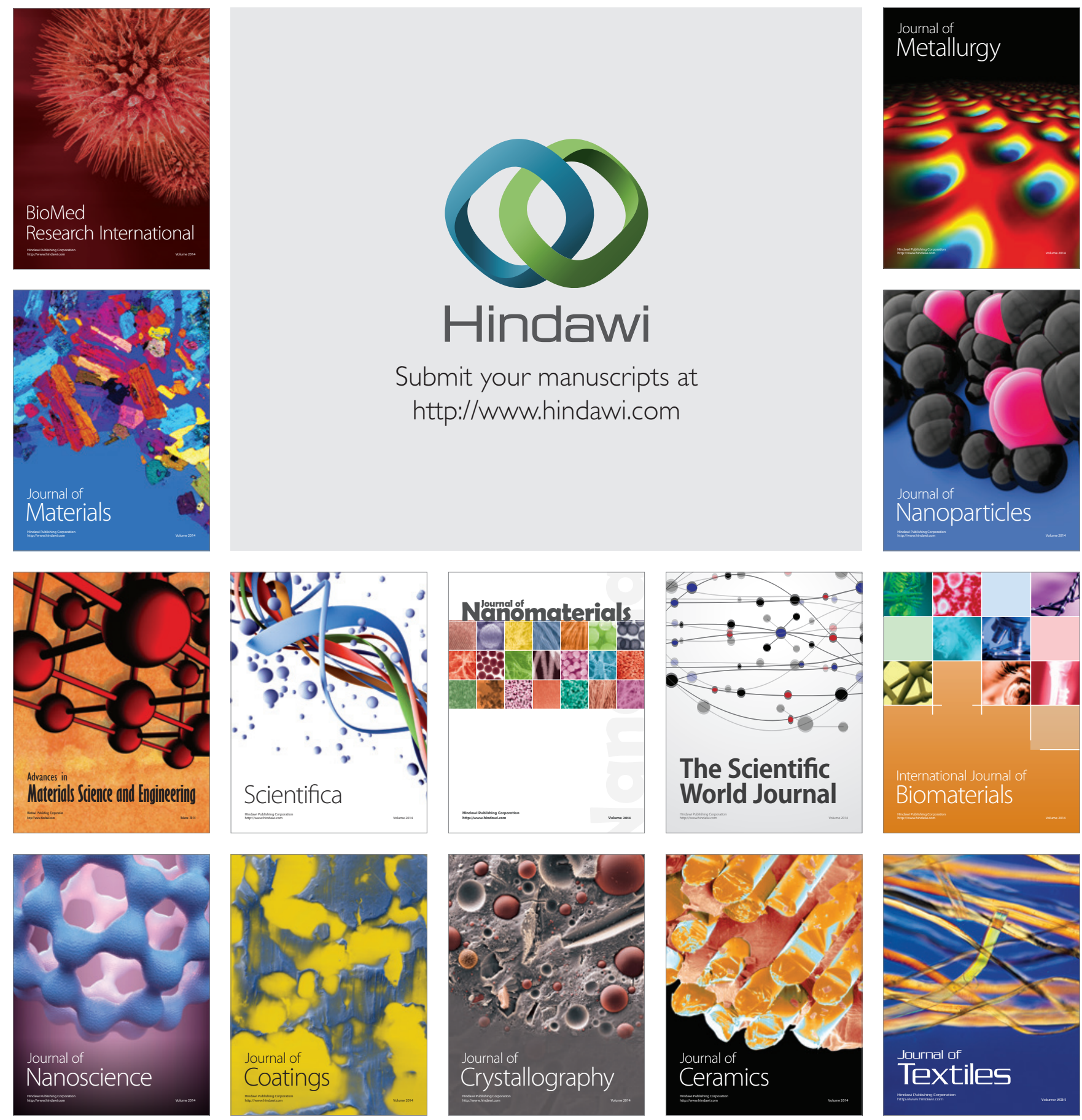